\title{
Misophonia: Evidence for an Elicited Initial Physical Response
}

\author{
Thomas H. Dozier ${ }^{1,2, *}$, Leighton Grampp ${ }^{3}$, Michelle Lopez ${ }^{1,3}$ \\ ${ }^{1}$ Misophonia Institute, Livermore, California, United States \\ ${ }^{2}$ Misophonia Treatment Institute, Livermore, California, United States \\ ${ }^{3}$ Department of Psychology, Alliant International University, San Diego, California, United States
}

Received May 11, 2020 ; Revised June 4, 2020; Accepted June 12,2020

Copyright (C2020 by authors, all rights reserved. Authors agree that this article remains permanently open access under the terms of the Creative Commons Attribution License 4.0 International License

\begin{abstract}
Misophonia is an understudied condition that is commonly viewed as an extreme sensitivity to specific soft sounds and visual stimuli which elicit strong negative emotions and physiological arousal. Recent research using self-report measures indicates that misophonia includes an elicited physical respondent (e.g. skeletal muscle flinch). Using electromyography (EMG) and direct observation, an immediate physical response to trigger stimuli was verified in three misophonic participants. Consistent movement of the body was observed in each subject when the subject reported having a moderate to strong response, though the location was unique for each subject. The response appeared to be immediate with the onset of a brief auditory stimulus and occurred at the start and throughout longer visual trigger stimuli. Additionally, an EMG measured response was consistent in one participant, and occurred in another participant when there was a strong self-reported response. Response latency for the EMG measured response was several hundred milliseconds, indicating the responses were elicited by the misophonic trigger stimulus and not general physiological arousal. This provides empirical evidence of an elicited physical response to misophonic stimuli in these individuals with misophonia. This supports conceptualization of misophonia as a condition which includes a respondent behavior (e.g. muscle flinch) elicited by the misophonic trigger stimulus. This highlights the need for additional research on the elicited physical response to misophonic stimuli and classical conditioning as a mechanism for the development of such a response.
\end{abstract}

Keywords Misophonia, Respondent Behavior, Classical Conditioning, Muscle Response, Human, Reflex

\section{Introduction}

Misophonia is an understudied and sparsely known condition characterized by extreme sensitivity to specific soft sounds (e.g. chewing, breathing) and/or visual images (e.g. chewing, hair twirling) that elicit strong emotion (e.g. anger, disgust, anxiety) and physiologic arousal (Dozier, 2015b; Dozier, Lopez, \& Pearson, 2017; Edelstein, Brang, Rouw, \& Ramachandran, 2013; Jastreboff \& Jastreboff, 2002; Kumar et al., 2017; Schröder, Vulink, \& Denys, 2013).

The misophonia response is generally considered to consist of extreme emotions elicited by the misophonic trigger stimuli and autonomic arousal as a stress response to the extreme emotions (Edelstein et al., 2013; Jastreboff \& Jastreboff, 2014; Kumar et al., 2017; Schröder et al., 2013; Wu, Lewin, Murphy, \& Storch, 2014). This is shown in Figure 1(a). An alternate view of misophonia proposes that misophonia also includes an initial physical reflex (e.g. muscle flinch) as shown in Figure 1(b) which has been presented in case studies and conference reports (Dozier, 2013, 2015a, 2015b, 2015c, 2017; Pearson 2015) and one experimental study (Dozier \& Morrison, 2017). 


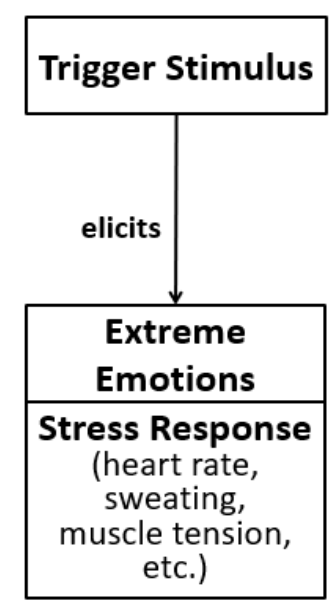

A

Figure 1. Composition of the misophonic response (a) as commonly reported in misophonia literature in which the trigger stimulus elicits extreme emotions and autonomic arousal, and (b) in which the trigger stimulus elicits a physical reflex and the combination of the physical reflex and trigger stimulus elicit extreme emotions and autonomic arousal

Dozier and Morrison (2017) exposed 26 misophonic individuals to their specific auditory and visual trigger stimuli and the individuals reported their immediate physical and emotional responses. Auditory and visual trigger stimuli were brief and low intensity so only a low-level response was experienced. All individuals reported an immediate physical response to trigger stimuli. The physical responses varied greatly among participants, with responses including muscle contraction sensation in shoulders, neck, hands/arms, jaw, back, thighs, toes, etc. Non-skeletal physical responses included warmth, nausea, and clitoral/vaginal sensations. A limitation of this study was reliance on self-report of participants and the lack of any temporal measure of the relationship between the trigger stimulus and the response.

The research goal of this pilot study was to expand on the findings of Dozier and Morrison (2017) to confirm the existence of an immediate physical response with direct observation of the response to misophonic stimuli, and to determine the temporal relationship between the misophonic stimulus and physical response. The methodology selected for this study was single subject design.

\section{Method}

\subsection{Subjects}

This research was reviewed and approved in advance by the IRB at Alliant International University, San Diego. Individuals interested in participating in the study completed a pre-study screening procedure by VSee internet video chat, during which they were exposed to weak triggers and asked to report on physical sensations and emotions experienced, as described in Dozier \& Morrison (2017). Eleven individuals were screened, and nine were eligible because they reported a muscle flinch response in a location that could be visually observed. The seven who were close to the research facility were invited to participate, but two declined due to schedule conflicts. Participants were at least 18 years of age and signed an informed consent. Participants were eligible if they experienced symptoms of misophonia, including a consistent, distinct physical response to a low intensity trigger stimulus, preoccupation with specific sounds, anger in response to trigger stimuli, efforts to avoid, and disruption in at least one life domain. Five participants participated in the initial test session. The three participants with the most distinct, observable physical responses were invited to participate in the second test session, which consisted of 2 women, Abby and Bonnie, ages 50 and 18, and one man, Dave, age 37 (names are pseudonyms). Control participants were eligible if they did not report any auditory or visual triggers, or other symptoms of misophonia on the Misophonia History Survey. Control participants were Caucasian, 2 women age 60 and one man, age 69.

\subsubsection{Misophonia History Survey}

This survey consisted of several misophonia severity surveys and identified the general categories of triggers (eating, breathing, etc.). Participant responses are listed in Table 1. 
Table 1. Participant Misophonia Survey

\begin{tabular}{|c|c|c|c|}
\hline Participant & Abby & Bonnie & Dave \\
\hline Age & 50 & 18 & 37 \\
\hline Gender & Female & Female & Male \\
\hline Race/Ethnicity & White/ Caucasian & White/ Caucasian & White/ Caucasian \\
\hline Medications currently taken & $\begin{array}{c}\text { levothyroxine } \\
\text { sodium, omeprazole, } \\
\text { diuretic }\end{array}$ & $\begin{array}{l}\text { venlafaxine, } \\
\text { bupropion }\end{array}$ & bupropion, sertraline \\
\hline \multicolumn{4}{|c|}{ Auditory Triggers } \\
\hline $\begin{array}{l}\text { Sounds of people eating - all forms of chewing, crunching, } \\
\text { smacking, swallowing, talking with food in mouth, etc. }\end{array}$ & Yes & Yes & Yes \\
\hline $\begin{array}{l}\text { Sounds of people drinking - sipping, slurping, saying “ah” } \\
\text { after a drink, swallowing, breathing after a drink, etc. }\end{array}$ & Yes & Yes & Yes \\
\hline $\begin{array}{l}\text { Sounds associated with eating - opening chip bags, water } \\
\text { bottle crinkling, setting a cup down, etc. }\end{array}$ & Yes & Yes & Yes \\
\hline $\begin{array}{c}\text { Other mouth sounds - sucking teeth, lip popping, kissing, } \\
\text { flossing, brushing teeth, etc. }\end{array}$ & Yes & Yes & Yes \\
\hline $\begin{array}{l}\text { Breathing sounds - sniffling, snorting, nasally breathing, } \\
\text { regular breathing, snoring, nose whistle, yawing, coughing, } \\
\text { throat clearing, hiccups, etc. }\end{array}$ & Yes & Yes & Yes \\
\hline Singing, humming, whistling & Yes & Yes & Yes \\
\hline $\begin{array}{l}\text { Work/school sounds - typing, mouse clicks, page flipping, } \\
\text { pencil on paper, copier sound, pen clicking, pen tapping, } \\
\text { tapping on desk, etc. }\end{array}$ & Yes & Yes & Yes \\
\hline $\begin{array}{c}\text { Animal sounds - dogs/cat grooming, dogs barking, rooster } \\
\text { crowing, birds chirping, crickets, frogs, animal scratching, } \\
\text { dog whimpering, etc. }\end{array}$ & Yes & Yes & Yes \\
\hline \multicolumn{4}{|c|}{ Visual Triggers } \\
\hline Jaw movement & Yes & Yes & Yes \\
\hline Open mouth chewing & Yes & Yes & Yes \\
\hline Repetitive hand movement such as twiddling thumbs & Yes & Yes & Yes \\
\hline $\begin{array}{l}\text { What emotional reactions do you usually have when you are } \\
\text { triggered? }\end{array}$ & anger, frustration & $\begin{array}{l}\text { anger, disgust, } \\
\text { suicidal thoughts }\end{array}$ & anger, disgust \\
\hline \multicolumn{4}{|c|}{ Do you experience any of the following as an immediate physical reaction to a single, strong trigger (auditory or visual)? } \\
\hline General muscle tension & No & Yes & Yes \\
\hline $\begin{array}{l}\text { Jerking, flinching, or twitching of specific skeletal muscles } \\
\text { such as shoulder, jaws, fists, toes, or neck }\end{array}$ & Yes & Yes & Yes \\
\hline Anxiety, panic, stress & Yes & Yes & Yes \\
\hline Misophonia Assessment Questionnaire Sum Score (0-63) & 32 (moderate) & 61 (extreme) & 53 (extreme) \\
\hline A-MISO-S (0-24) & 11 (moderate) & 23 (extreme) & 15 (severe) \\
\hline
\end{tabular}

\subsubsection{Misophonia Assessment Questionnaire (MAQ)}

The MAQ is a self-report measure that contains 21 items (Dozier, 2017). Example items include "My sound issues currently create problems for me" and "My sound issues currently make me feel helpless." Items are rated on a 4-point scale that ranges from 0 (not at all) to 3 (almost all the time). Total score can range from 0 to 63 .

\subsubsection{Amsterdam Misophonia Scale (A-Miso-S)}

The A-Miso-S is a self-report measure adapted from the Yale-Brown Obsessive-Compulsive Scale (Schröder et al., 2013). Total score can range from 0 to 24 .

\subsubsection{Surface Electromyography System (EMG)}

Muscle response was recorded using J\&J Engineering I-330-C2+ hardware and J\&J Use3 software for windows. EMG responses are measured in microvolts $(\mu \mathrm{V})$ and displayed graphically.

\subsection{Apparatus}

The study was conducted in a psychologist's office in a private practice clinic in San Diego, CA. Equipment for the study consisted of two Windows laptop computers, Samsung Galaxy S3 smartphone, Bose QC25 headphones, a speaker, a portable massage table, 19-inch LCD television (Element model ELEFW195), and J\&J Engineering I-330-C2+12 system with four EMG channels. Three channels of the EMG system were connected to 
electrodes placed on the subjects to measure muscle action. One channel was connected to the stimulus presentation system (computer or smartphone) to record the timing.

Auditory stimuli were prerecorded and stored on one computer. Stimuli were played using Sony Sound Forge Audio Studio software, which allowed control of the stimulus duration. The left audio output was split, and connected to Bose QC25 noise cancelling headphones for the subject and to a speaker for the researchers. The right audio channel was acoustically coupled to an EMG input channel. The EMG input contained a low-pass noise filter, so some trigger stimuli (e.g. sniffing) were completely blocked by the filter. A $100 \mathrm{~ms}$ duration, $150 \mathrm{~Hz}$ timing pulse was added to the right channel immediately preceding the trigger stimulus. This provided both synchronization and a time-base reference for data recording.

Auditory trigger stimuli were either common trigger stimuli which had previously been recorded by the first author (sniffing, chewing) or recorded from YouTube (dog barking). Neutral stimuli were sounds recorded from the application White Noise by TMSoft and included train clacking, gulf waves, frogs croaking, and room of people talking. The duration for neutral stimuli was 1.0s. The duration for the trigger stimuli varied from $0.38 \mathrm{~s}$ to $0.83 \mathrm{~s}$ based on the testing in the first session. The volume of each auditory stimulus was adjusted using the audio editor to a power of -20 db RMS. The volume setting on the computer was set at $10 \%$, which was a soft, easily audible level.

The visual stimuli were recorded on smartphones. One was the family dog licking a pillow. The other two visual triggers were of a man chewing. The neutral stimuli included a puppy doing tricks, a man rubbing his face with his hand, and a man reading a product box (moving his head and hands). All visual stimuli were presented with 3.0s duration. The Visual Trigger Tamer ${ }^{\circledR}$ application was used to present the stimuli with the smartphone. The television was placed 20 inches in front of the participant's face, with the participant lying face down on the massage table viewing the screen through the face opening of the table head support. The visual trigger image was 16:9 aspect ratio, 8.5-inch diagonal. A white "+" was shown in the center of the screen preceding the trigger video clip. The application provided control of the duration of stimuli. An auditory timing pulse was added to the start of each visual stimulus for synchronization with the EMG response. The audio was connected to the EMG system, but the subject wore headphones without connection for sound isolation.

For all stimuli, the output of the EMG system was graphically displayed on the second computer and the data was captured continuously as a video recording using Snagit ${ }^{\circledR}$ software.

\subsection{Procedure}

The study consisted of two sessions with each participant. The purpose of the first session was to determine electrode location for recording the EMG and physical movement response to trigger stimuli. Various locations for the electrodes were tested based on the participant comments and visual observation of the researchers. During the first visit, the participant laid face down on a massage table while several sets of surface electrodes were attached to muscles. Each participant was exposed to recorded auditory and visual trigger stimuli which had previously been identified as stimuli which evoked a misophonic response. The electrode locations were varied in an attempt to record an EMG response. The physical and emotional responses to each trigger were reported on a scale of $0-10$. The duration of the trigger stimulus was initially short, and was increased until a rating of 1-4 was achieved for the physical and/or emotional response. The location of the electrodes with the highest response was recorded for use in the second session. This procedure was repeated for a total of 3 triggers (2 auditory and 1 visual).

The purpose of the second session was to submit each participant to trigger and neutral stimuli, while measuring EMG of the same 3 muscle groups on each individual and visually observing the participant for a physical response to stimuli. Two unique auditory stimuli were selected for each participant. A total of 4 unique triggers were used, which included chewing sounds, sniffing, and dog barking; and 4 neutral auditory stimuli were used. One unique visual stimulus was selected for each participant. A total of 3 unique trigger stimuli were used, which included open mouth chewing, jaw movement while chewing, and a dog licking a pillow. The timing pulse on the videos functioned properly in session 1 , but was inoperable in session 2 . The participants were exposed to only the visual element of the trigger visual stimulus.

Non-invasive surface electromyography (EMG) leads were attached with gel electrodes where responses had been recorded in session 1. Electrode placement was the same for all participants in session 2. EMG electrodes were placed on the right posterior deltoid, T-2 paraspinals, and lateral gastrocnemius for each participant. EMG output to the computer screen was captured while the participant was exposed to the complete set of recorded trigger and neutral stimuli.

The auditory stimuli were presented first in random order with each stimulus presented 3 times. Visual stimuli were presented second in random order with each stimulus presented 3 times. The name of the stimulus was communicated to the participant before each presentation. After every stimulus presentation, the participant rated the severity of the physical response (0-10 scale) and the emotional distress (0-10 scale), where 0 was no perceived physical response and 10 was the maximum real-life response to trigger stimuli. Participants were instructed to rest and relax their muscles between triggers. Auditory stimuli were presented at a rate of 2 per minute and visual 
stimuli at a rate of 1 per minute. Participants were observed by two researchers when exposed to stimuli. When muscle movement was limited to one area (such as upper back), both observers viewed the same area. When the muscle movement was in different areas (e.g. calf and back), each observer viewed a different area. Observers conferred with simultaneous hand signals of the severity of the observed response (0-3 rating). Agreement was $100 \%$ on the presence/absence of the response when observing the same area.

\section{Results}

\subsection{Observed Physical Response}

A physical response was observed on each participant. The observed responses resembled a tic-like muscle contraction on the back of the neck for Dave and in the middle of the back for Abbie. The physical response for Bonnie resembled a muscle contraction on her calves and shoulder blades.

Figure 2 shows the relationship of the severity of physical response reported by the participant and the rate of an observed physical response to all auditory trigger, visual trigger, and neutral stimuli. In total, there were 55 reports of a physical response and 30 instances of an observed response. Physical responses were never observed when the participant did not report a physical response. There were no observed physical responses in the control participants to any of the stimuli.

Table 2 shows the responses to each participant's individual trigger stimuli. One neutral stimulus was a "room of people." Abby reported a weak physical response to each instance of this stimulus and Bonnie reported a weak response to one instance of it. Dave reported a weak response to one instance of the neutral stimulus, "gulf waves."

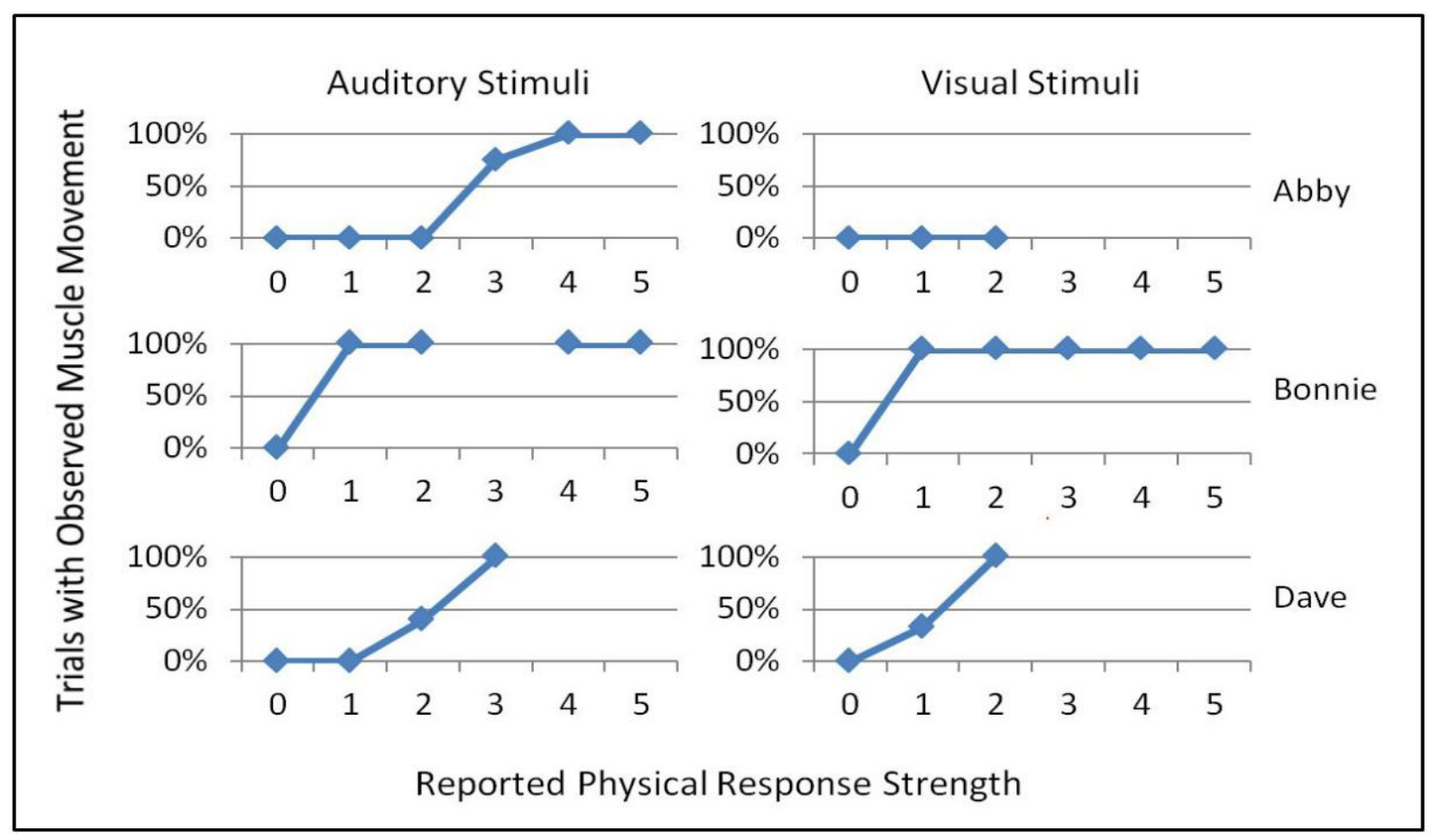

Figure 2. Instance of observed physical response (visible muscle/tissue movement) versus the participant self-report severity of physical response to all auditory and visual stimuli.

Table 2. Rate of physical response to auditory stimuli.

\begin{tabular}{|c|c|c|c|c|c|c|}
\hline & \multicolumn{2}{|c|}{ Auditory Personal Trigger Stimuli (N=6) } & \multicolumn{3}{c|}{$\begin{array}{c}\text { Auditory Neutral Stimuli } \\
\text { (N=12) }\end{array}$} \\
\hline & Self Report & Observed & EMG Response & Self Report & Observed & EMG Response \\
\hline Abby & $100.0 \%$ & $66.7 \%$ & $16.7 \%$ & $25.0 \%$ & $0.0 \%$ & $0.0 \%$ \\
\hline Bonnie & $100.0 \%$ & $100.0 \%$ & $100.0 \%$ & $8.3 \%$ & $0.0 \%$ & $0.0 \%$ \\
\hline Dave & $100.0 \%$ & $50.0 \%$ & $0.0 \%$ & $8.3 \%$ & $0.0 \%$ & $0.0 \%$ \\
\hline Control group* & $2.8 \%$ & $0.0 \%$ & $0.0 \%$ & $0.0 \%$ & $0.0 \%$ & $0.0 \%$ \\
\hline
\end{tabular}

* Each of the 3 control participants was exposed to 12 trigger stimuli and 6 neutral stimuli. 
Table 3 shows the response of each participant to their own visual trigger stimulus and to neutral stimuli. One of the neutral stimuli was a person rubbing his face, which triggered a physical and anger response for Abby.

\subsection{EMG Response}

Although the authors observed a physical response to trigger stimuli for each participant, we were unable to locate electrode placement for an EMG response on Abby and Dave during the first visit. Bonnie had a consistent, strong calf muscle response.

EMG response was measured as present or absent. To qualify as an EMG response, the muscle activity had to show a visually unique pattern compared to 1 s before and 1s after the trigger stimulus.

For Bonnie, an EMG response was detected to each instance of all misophonic stimuli in which she reported a physical or emotional response. The EMG response was detected $100 \%$ on her calf (channel D), $77.8 \%$ on her deltoid (channel B), and $27.8 \%$ on her paraspinals (channel C.) Greater consistency of the muscle responses is evident by classifying the responses by type of trigger stimulus (Table 4). Bonnie's EMG response to dog licking visual stimulus was only the calves.

Figure 3 shows a representative EMG response on channel D (calf muscle), and channel B (deltoid), when exposed to the auditory stimulus of a sniff.

Table 3. Rate of physical response to visual stimuli.

\begin{tabular}{|c|c|c|c|c|c|c|}
\hline & \multicolumn{2}{|c|}{ Visual Personal Trigger Stimuli (N=3) } & \multicolumn{3}{c|}{ Visual Neutral Stimuli (N=9) } \\
\hline & Self Report & Observed & EMG Response & Self Report & Observed & EMG Response \\
\hline Abby & $100.0 \%$ & $0.0 \%$ & $0.0 \%$ & $33.3 \%$ & $0.0 \%$ & $0.0 \%$ \\
\hline Bonnie & $100.0 \%$ & $100.0 \%$ & $100.0 \%$ & $0.0 \%$ & $0.0 \%$ & $0.0 \%$ \\
\hline Dave & $66.7 \%$ & $33.3 \%$ & $0.0 \%$ & $0.0 \%$ & $0.0 \%$ & $0.0 \%$ \\
\hline Control group* & $0.0 \%$ & $0.0 \%$ & $0.0 \%$ & $0.0 \%$ & $0.0 \%$ & $0.0 \%$ \\
\hline
\end{tabular}

* Each of the 3 control participants was exposed to 9 trigger stimuli and 9 neutral stimuli.

Table 4. Muscle responses of Bonnie for different types of trigger stimuli.

\begin{tabular}{|c|c|c|c|}
\hline Muscle & Deltoids & Paraspinals & Calves \\
\hline All Auditory Triggers (N=9) & $8(88.9 \%)$ & $1(11.1 \%)$ & $9(100 \%)$ \\
\hline Visual, Eating (N=6) & $6(100 \%)$ & $4(66.7 \%)$ & $6(100 \%)$ \\
\hline Visual, Dog Licking (N=3) & $0(0 \%)$ & $0(0 \%)$ & $3(100 \%)$ \\
\hline
\end{tabular}

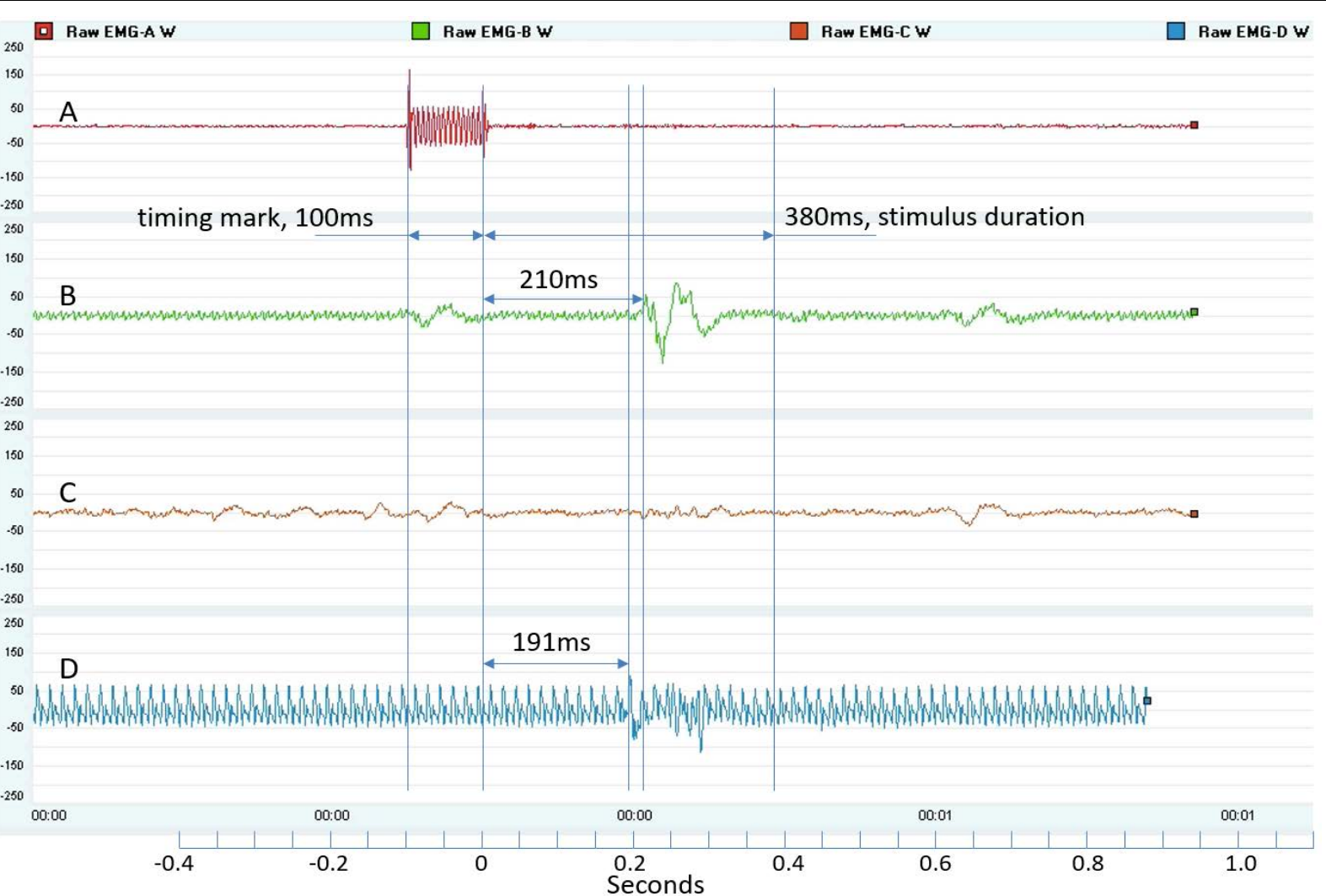

Figure 3. EMG response for Bonnie for stimulus sniff-little. Channel A: inaudible timing pulse. Channel B: deltoid muscle. Channel C: paraspinal muscle. Channel D: calf muscle. 


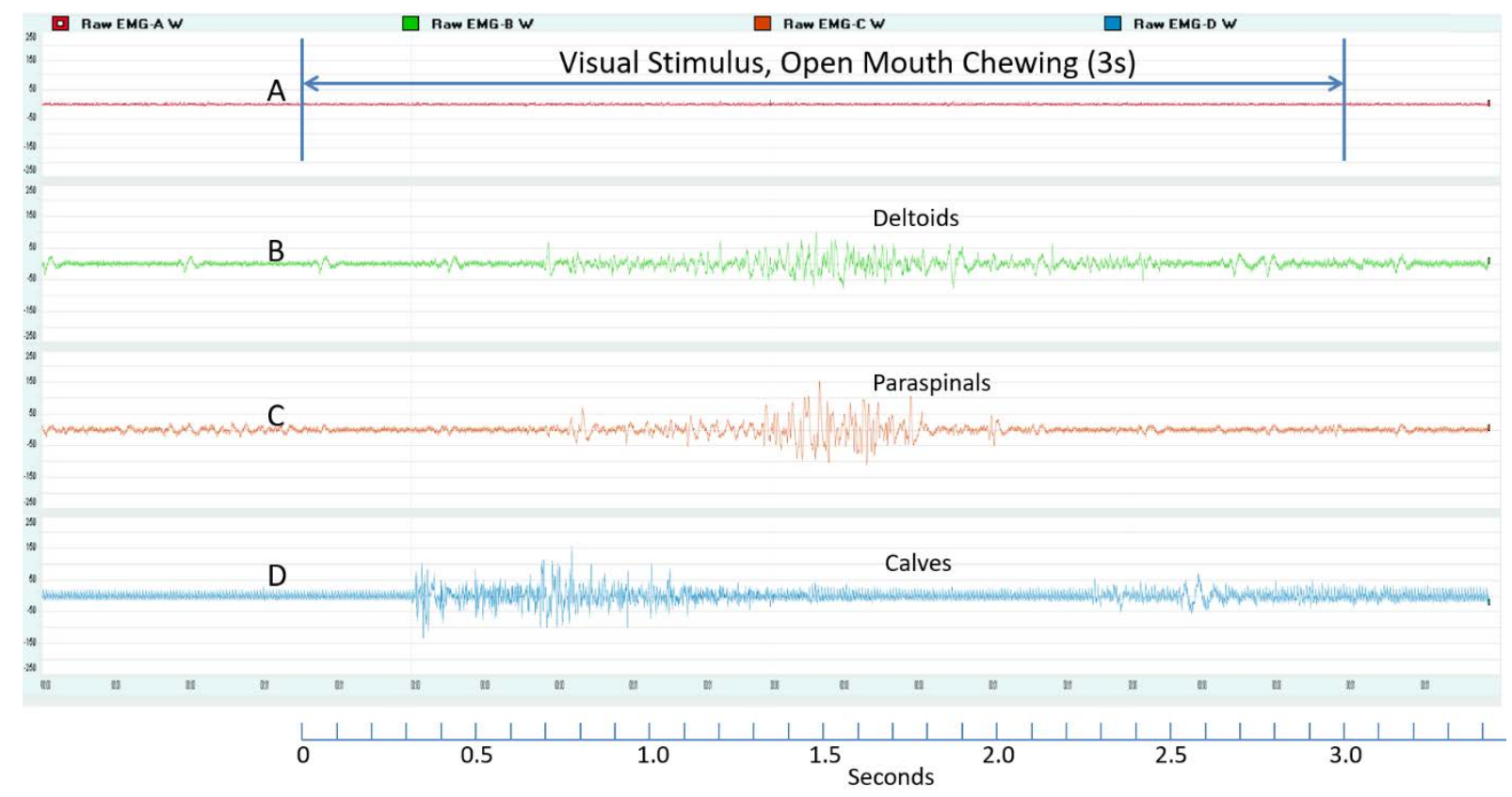

Figure 4. EMG response for Bonnie for stimulus open-mouth-chewing.

Figure 4 shows a representative EMG response of Bonnie on all three measurement channels to a 3s visual trigger stimulus, open-mouth-chewing.

Abby showed an EMG response on the deltoid muscle only when she reported a physical response of 4 or higher, which occurred twice. There was no EMG response when her response severity rating was lower. Dave did not demonstrate an EMG response to any of the reported auditory or visual stimuli presented. There were no EMG responses to any stimuli for any of the control participants.

\subsection{Response Latency}

The average response latency for auditory stimuli for Bonnie was 195 ms, (minimum 106 ms, maximum 352 ms, $\mathrm{N}=9$ ). For visual stimuli with data from the initial session, the average response latency was $301 \mathrm{~ms}$ (minimum 173, maximum 392, $\mathrm{N}=4$ ). The response latency for auditory stimuli for Abby was $166 \mathrm{~ms}$ (152 ms, 172ms, N=2).

\section{Discussion}

Although a muscular response elicited by misophonic stimuli has been anecdotally and self-reported, this is the first study to verify a muscular reflex in a person with misophonia using direct observation. Visible physical movement when exposed to auditory misophonic stimuli was observed on each participant. When the participant reported a response severity to auditory or visual stimuli of 4 or higher (scale of $0-10$ ), the researchers observed immediate physical movement $100 \%$ of the time (see Figure2). The researchers observed the physical movement as an immediate response to auditory stimuli with no perceptible delay. Auditory stimuli were brief, eliciting a single flinch. Visual stimuli were 3 seconds duration and elicited immediate and longer duration responses as observed by the researchers. Therefore, the objective of independent verification of an immediate physical response to trigger stimuli was accomplished.

The EMG response on one of the three participants was recorded for all trials in which she reported feeling a physical response. An EMG response was recorded on a second participant when self-report rating of physical response was high. The typical stimulus-response latency was 150 to $200 \mathrm{~ms}$ for auditory stimuli. The short response latency implies that the physical misophonic responses measured in this study were respondent behavior elicited by the misophonic stimuli, and not a result of evaluative mental processes or a stress response from the extreme emotions.

Although there was consistent visible movement in the back or neck in response to trigger stimuli for 2 participants, varied placement of the electrodes did not yield a consistent EMG response. The authors postulate that there was a muscle action causing the visible movement that was either too deep to be detected by surface EMG or the muscle which was activated was not in our view. For example, the observed movement on the back of the neck may have been the result of muscles tensing on the front of the neck or chest.

Consistent with the findings of Dozier and Morrison (2017), the physical response was different for each participant. One participant had a visible response on his neck and another a visible response on her back. The third participant had a consistent EMG and visual calf muscle response, but the complete response was different for auditory and visual stimuli, with low incidence of response 
of paraspinals to auditory stimuli, and a high incidence to visual eating-related stimuli. Even more striking was the absence of deltoids or paraspinals responses for a recently acquired visual trigger of the participant's dog licking a pillow, which activated only her calf muscles. These highlights both the similarities of auditory and visual misophonic responses, and the peculiar differences of misophonic responses for various stimuli, within and between misophonic individuals. The existence of a unique elicited muscle response in this study and Dozier and Morrison (2017) may support classical conditioning as a mechanism for the etiology of misophonia.

\section{Theoretical Implications}

Stimulus-response classical conditioning (Donahoe and Vegas, 2004) occurs when there is temporal pairing of a neutral stimulus and a muscle response. This needs further research for the development of muscle responses to common stimuli, when there is no apparent unconditioned stimulus for the response. A common example would be a shoulder muscle flinch elicited by a surgeon's pager (E. Lipov, personal communication, May 17, 2018). An elicited muscle flinch from a phone ringtone has also been reported by a professional receiving stressful phone calls, and a cancer patient reported a lower back muscle flinch to the sound and vibration of her portable chemotherapy pump (Dozier, 2017).

We posit that the muscle responses elicited by misophonic stimuli demonstrated in the participants of this study are conditioned aversive respondents, and such respondent behavior may be an important component in the development and maintenance of the overall misophonic response. Further study is warranted on the initial physical responses elicited by misophonic stimuli and on stimulus-response classical conditioning as a fundamental neurological principle.

\section{Limitations}

Several study limitations should be noted. First, individuals were selected because of their self-report of a physical response to trigger stimuli, therefore generalizability is limited. Second, the latency of the physical response to stimuli for one participant could not be verified due to an inability to measure their response with EMG. Third, participants were informed of the stimulus prior to exposure, which may have affected their response. For example, during the first session, one participant was not informed of the change from a trigger stimulus (sniffing) to a similar but neutral stimulus (gulf waves). The participant had a trigger response to the neutral stimulus the first time, but did not have a response to a second instance of the stimulus, which suggests that expectation may affect the misophonic response. Fourth, psychometric properties of the MAQ and A-Miso-S misophonia severity instruments have not been established. Fifth, a diagnostic assessment was not administered. Rather, participants reported on diagnoses they had received in the past. Future studies should include a clinician-administered diagnostic interview, which could provide pertinent information about the clinical presentation of misophonia and co-occurring diagnoses. Sixth, EMG was measured by a response being 'present' or 'absent' rather than quantifiably. In addition, surface EMG has some limitation due to the inability to detect deep muscle movement. Future studies would benefit from mathematical calculation of EMG stimulus strength and could consider needle EMG to detect both smaller movements and movements deeper within muscle tissue.

\section{Conclusions}

This study provides empirical evidence of an elicited physical response to misophonic stimuli in three individuals with misophonia, with the response latency of about $200 \mathrm{~ms}$ for auditory stimuli. This provides support for the conceptualization of misophonia as a condition which includes a respondent behavior (e.g. muscle flinch) elicited by the misophonic trigger stimuli. The authors emphasize that the observed physical responses and EMG data identified in this study are different from skin conductance responses and general muscle tension reported in previous studies. This highlights the need for additional research on the elicited physical response to misophonic stimuli and classical conditioning as a mechanism for the development of such a response.

\section{Ethics Approval and Consent to Participate}

Alliant International University Institutional Review Board provided approval for ethics and consent for participate.

\section{Human and Animal Rights}

The procedures followed were in accordance with the ethical standards of Alliant International University Institutional Review Board. No animals were used in this study.

\section{Conflict of Interest}

The authors declare no conflict of interest. 


\section{REFERENCES}

[1] Bernstein, R. E., Angell, K. L., \& Dehle, C. M. (2013). A brief course of cognitive behavioural therapy for the treatment of misophonia: A case example. The Cognitive Behaviour Therapist, 6, 1-13. doi:10.1017/S1754470X1300 0172

[2] Cohen, J. (2011). When a chomp or a slurp is a trigger for outrage. The New York Times, 5 September 2011 (http://www.nytimes.com/2011/09/06/health/06annoy.html? r=1\&).

[3] Donahoe \& Vegas (2004). Pavlovian Conditioning: The CS-UR Relation. Journal of Experimental Psychology: Animal Behavior Processes, 30(1), 17-33. doi:10.1037/0097-7403.30.1.17

[4] Dozier, T. H. (2013). Misophonia Reflexes and Treatment. Presentation at the 1st Misophonia Association Conference; 2013 Oct 25-26; Portland. OR, USA. Available from: https://misophoniainstitute.org/wp-content/uploads/2017/07 /Dozier-2013-Misophonia-Reflexes-and-Treatment.pdf

[5] Dozier, T. H. (2015a). Counter Conditioning treatment for misophonia. Clinical Case Studies, 14, 1-14. doi; $10.1177 / 1534650114566924$

[6] Dozier, T. H. (2015b). Etiology, composition, development and maintenance of misophonia: A conditioned aversive reflex disorder. Psychological Thought, 8, 1-16. doi: 10.5964/psyct.v8i1.132

[7] Dozier, T. H. (2015c). Treating the initial physical reflex of misophonia with the neural repatterning technique: A counterconditioning procedure. Psychological Thought, 8(2), 189-210. doi:10.5964/psyct.v8i2.138

[8] Dozier-T. H. (2017). Understanding and overcoming misophonia: A conditioned aversive reflex disorder. 2nd ed. Livermore (CA): Misophonia Treatment Institute.

[9] Dozier, T. H., \& Morrison, K. L. (2017). Phenomenology of misophonia: initial physical and emotional responses. American Journal of Psychology, 130(4), 431-438. doi: 10.5406/amerjpsyc.130.4.0431

[10] Edelstein, M., Brang, D., Rouw, R., \& Ramachandran, V.S. (2013). Misophonia: Physiological investigations and case descriptions. Frontiers in Human Neuroscience, 7(296), 1-11. doi: 10.3389/fnhum.2013.00296

[11] Jastreboff, M. M., \& Jastreboff, P. J. (2002). Decreased sound tolerance and tinnitus retraining therapy (TRT). The Australian and New Zealand Journal of Audiology, 24(2), 74-84. doi:10.1375/audi.24.2.74.31105

[12] Jastreboff, M.M., \& Jastreboff, P.J. (2014). Treatments for decreased sound tolerance (hyperacusis and misophonia). Seminars in Hearing 35(2), 105-120. doi: 10.1055/s-0034-1372527

[13] Kumar, S., Tansley-Hancock, O., Sedley, W., Winston, J. S., Callaghan, M. F., Allen, M., ... \& Griffiths, T. D. (2017). The brain basis for misophonia. Current Biology, 27(4), 527-533. doi: 10.1016/j.cub.2016.12.048

[14] Pearson, C (2015). A new hypnotherapy for misophonia. Presentation at the 3rd Misophonia Association Conference; 2015 Oct 16-17; Chicago, IL, USA. Available from: https://misophoniainstitute.org/wp-content/uploads/2017/07 /Pearson-2015-Sequent-Repatterning.pdf

[15] Schröder, A., Vulink, N., \& Denys, S. (2013). Misophonia: Diagnostic criteria for a new psychiatric disorder. PLoS ONE 8, e54706. doi: 10.1371/journal.pone.0054706

[16] Wu, M. S., Lewin, A. B., Murphy, T. K., \& Storch, E. A. (2014) Misophonia, incidence, phenomenology, and clinical correlates in an undergraduate student sample. Journal of Clinical Psychology, 70, 994-1007. doi: 10.1002/jclp.22098 